\title{
Double screw-sense inversions of helical chiral-achiral random copolymers of fluorene derivatives in phase separating solutions
}

\author{
Yusuke Sanada ${ }^{1}$, Ken Terao and Takahiro Sato \\ The addition of methanol to dilute THF solutions of chiral-achiral random copolymers of fluorene derivatives and the chiral \\ homopolymer showed thermo-reversible circular dichroism (CD) induction in the main-chain fluorene absorption region. \\ This finding demonstrated the uneven population of the right- and left-handed helical conformations in the polymer chains. \\ From the sign of the induced $C D$, two helical screw-sense inversions were found by changing the chiral monomer content. \\ The Ising model for chirally interacting chiral-achiral random copolymers can explain the double screw-sense inversions. \\ Polymer Journal (2011) 43, 832-837; doi:10.1038/pj.2011.75; published online 3 August 2011
}

Keywords: chiral-achiral random copolymer; circular dichroism induction; helical screw-sense inversion; phase separation; polyfluorene

\section{INTRODUCTION}

Recently, $\pi$ - and $\sigma$-conjugated polymers, which possess interesting electrical and optical properties, have attracted much interest as candidates for organic light-emitting diodes ${ }^{1-3}$ and chemical sensors. ${ }^{4}$ Chiroptical properties, such as circular dichroism (CD), optical rotation, and circular polarized luminescence, enrich the applications of these organic semiconductors as optical devices or sensors. ${ }^{5-9}$ Optically active side chains may introduce chiroptical properties into conjugated polymers, but some polymers exhibit these properties only in films or phase separating solutions, ${ }^{10-13}$ which indicates that intermolecular chiral interactions are necessary to induce the chiroptical properties in such conjugated polymers.

Polyfluorene derivatives are $\pi$-conjugated polymers. From electron and X-ray diffraction, ${ }^{14,15}$ as well as molecular modeling, ${ }^{14,} 16$ these polymers are known to take a helical conformation, but chirality introduced to the side chain of the polymers was not found to induce circular dichroism (CD) on their backbone chain in dilute solution. This finding probably results from weak intramolecular chiral interactions that cannot discriminate against the handedness of the backbone helical conformation.

In a previous study, ${ }^{17}$ the $\mathrm{CD}$ induction of an optically active homopolymer of fluorene derivative, poly(2,7-[9,9-bis((S)-citronellyl)]fluorene) (PCF), in dilute THF solutions was found by the addition of a non-solvent methanol. Although the methanol-added solutions were almost transparent because of low polymer concentrations $\left(\sim 10^{-6} \mathrm{~g} \mathrm{~cm}^{-3}\right)$, static light scattering measurements indicated a liquid-liquid phase separation to form concentrated droplet phases, and it was concluded that this induction arises from the intermolecular chiral interaction of polyfluorene chains in the concentrated droplet phases separated from the dilute solution.

Helical chiral-achiral random copolymers often exhibit interesting chiroptical properties, for example, sergeants-and-soldiers behavior, ${ }^{18-20}$ composition-driven helical screw-sense inversion, ${ }^{21,22}$ and so on. Therefore, the CD induction study in phase separating solution was recently extended to a helical chiral-achiral random copolymer of fluorene derivatives, poly(2,7-[9,9-bis((S)-citronellyl)]fluorene-random-9,9-di-n-octyl fluorene), as shown in Scheme 1, and double screw-sense inversions were found by changing the chiral monomer content $x$. To the best of our knowledge, this is the first observation of composition-driven double sense inversions in helical chiral-achiral random copolymers. In this paper, this finding is reported along with the theoretical argument of its origin.

\section{EXPERIMENTAL PROCEDURE}

\section{Samples}

Mixtures of the chiral and achiral dibromofluorene monomers of different compositions were copolymerized in a hot mixture of toluene and $\mathrm{N}, \mathrm{N}$-dimethylformamide (DMF) using a zero-valent nickel reagent by the Yamamoto coupling reaction. ${ }^{7}$ Each copolymer sample was divided into three fractions by fractional precipitation using THF as the solvent and methanol as the precipitant, and the middle main fraction was chosen for the following experiments.

The chiral monomer content $x$ of five middle copolymer fractions was estimated from the ratio of the integrated intensities of the proton signals of the chiral side chains and aromatic rings. The results, listed in Table 1, are very

Department of Macromolecular Science, Osaka University, Osaka, Japan

${ }^{1}$ Current address: Department of Chemical Processes and Environments, Faculty of Environmental Engineering, The University of Kitakyushu, 1-1 Hibikino Wakamatsu-ku, Kitakyushu, Fukuoka 808-0135, Japan

Correspondence: Professor T Sato, Department of Macromolecular Science, Osaka University, 1-1 Machikaneyama-cho, Toyonaka, Osaka 560-0043, Japan.

E-mail: tsato@chem.sci.osaka-u.ac.jp

Received 9 April 2011; revised 4 June 2011; accepted 8 June 2011; published online 3 August 2011 


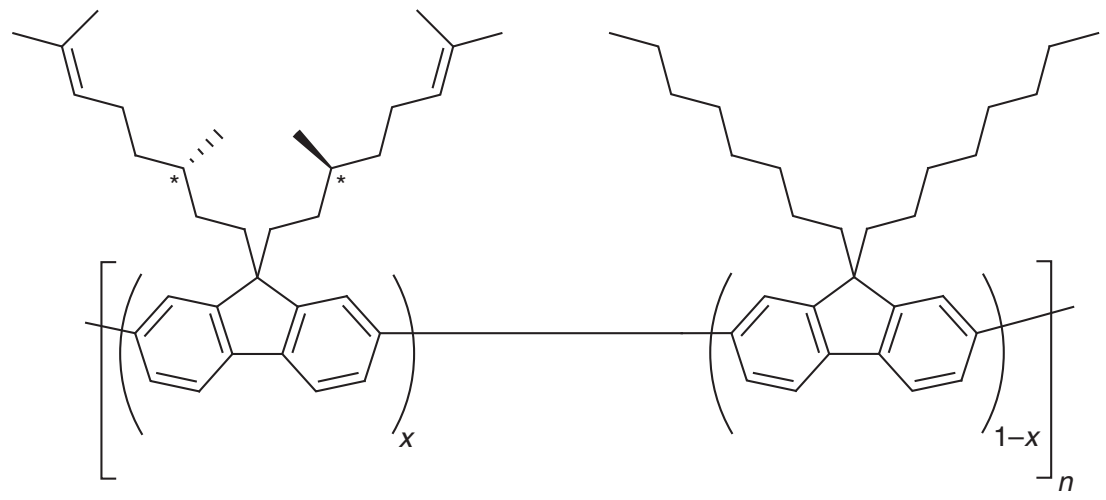

Scheme 1 Chemical structure of the chiral-achiral random copolymer studied.

Table 1 Characteristics of polymer fractions used in this study and of concentrated droplet phases in methanol-added THF solutions of fractions PC505 and PCF

\begin{tabular}{|c|c|c|c|c|c|c|c|}
\hline Fraction & $x$ & $M_{\mathrm{w}} / 10^{4}$ & $N_{0, w}$ & $M_{\mathrm{w}} / M_{\mathrm{n}}^{\mathrm{a}}$ & $R_{\mathrm{w}} / \mathrm{nm}^{\mathrm{b}}$ & $R_{\mathrm{w}} / R_{\mathrm{n}}^{\mathrm{b}}$ & $c_{\mathrm{c}} \mathrm{gcm}^{-3_{\mathrm{b}}}$ \\
\hline PC802 & $0.80(0.8)^{c, d}$ & $11.5^{\mathrm{a}}$ & 267 & 1.5 & & & \\
\hline PC604 & $0.60(0.6)^{c, d}$ & $4.1^{a}$ & 98 & 1.3 & & & \\
\hline PC505 & $0.50(0.5)^{c, d}$ & $7.0^{\mathrm{a}}$ & 169 & 1.4 & $110^{e}(83)^{f}$ & $1.4^{e}(1.3)^{f}$ & $0.60^{e}(0.59)^{f}$ \\
\hline PC208 & $0.19(0.2)^{c, d}$ & $10.5^{a}$ & 264 & 1.5 & & & \\
\hline PCF & $1.0(1.0)^{\mathrm{d}}$ & $13.4^{\mathrm{a}}$ & 305 & - & $150^{e}(170)^{f}$ & $1.8^{e}(1.8)^{f}$ & $0.50^{e}(0.50)^{f}$ \\
\hline
\end{tabular}

aDetermined by SEC-MALS

betermined by SLS.

'Determined by $1 \mathrm{H}$ NMR.

${ }^{d}$ Chiral monomer initial composition fed at the polymerization.

eat $15^{\circ} \mathrm{C}$.

fat $40^{\circ} \mathrm{C}$.

close to the initial compositions fed at the copolymerization (the numbers in the parentheses in the second column of Table 1). The weight average molecular weights, $M_{\mathrm{w}}$, and the ratios of $M_{\mathrm{w}}$ to the number average, $M_{\mathrm{n}}$, of the five fractions were determined by size-exclusion chromatography with a multi-angle light scattering detector (SEC-MALS). ${ }^{23}$ Table 1 lists the results and weight average degree of polymerization $N_{0, \mathrm{w}}$ calculated from the $M_{\mathrm{w}}$ determined. The values of $M_{\mathrm{w}} / M_{\mathrm{n}}$ indicate the relatively narrow molecular weight distributions of the fractions used in this study. One fraction of the chiral homopolymer PCF prepared in the previous study was added to the copolymer fractions for the following CD induction study, and its $M_{\mathrm{w}}$ was determined by static light scattering (SLS). ${ }^{17}$ The values of $N_{0, w}$ of all the fractions were within the range of ca. 100-300.

\section{Measurements}

Each fraction was first dissolved in THF $\left(8 \times 10^{-7} \mathrm{~g} \mathrm{~cm}^{-3}\right)$, and then, methanol was added to the THF solution at room temperature. The methanol volume fraction $\phi_{\mathrm{MeOH}}$ in the mixed solvent and the final polymer concentration $c$ in the THF-methanol solution were adjusted to 0.5 and $4 \times 10^{-7} \mathrm{~g} \mathrm{~cm}^{-3}$, respectively. CD and SLS measurements were conducted using the THF-methanol solutions prepared using a JASCO J-720WO spectropolarimeter and a Fica 50 light scattering photogoniometer with $546 \mathrm{~nm}$ incident light, respectively, at 40 and $15^{\circ} \mathrm{C}$.

\section{RESULTS}

\section{Static light scattering}

Figure 1 shows the light scattering profiles of THF-methanol solutions of fractions PC5O5 $(x=0.50)$ and PCF $(x=1)$ at $40{ }^{\circ} \mathrm{C}$ and $15^{\circ} \mathrm{C}$ (300 min after quenching from $40^{\circ} \mathrm{C}$ ). Here, $K$ is the optical constant, $c$ is the polymer mass concentration, $R_{\theta}$ is the excess Rayleigh ratio at the scattering angle $\theta$, and $k^{2}$ is the square of the scattering vector. All of the profiles exhibit very strong angular dependences, demonstrating the existence of large particles in the solutions. The phase diagram obtained previously ${ }^{17}$ for the methanol-added THF solution of

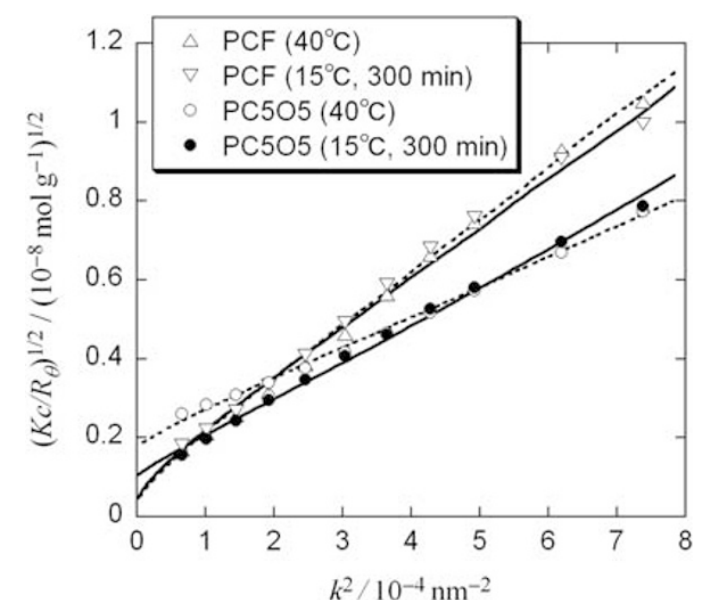

Figure 1 Berry plots for methanol-added THF solutions of fractions PC505 $(x=0.50)$ and $\mathrm{PCF}(x=1)$ at $40^{\circ} \mathrm{C}$ and $15^{\circ} \mathrm{C}(300 \mathrm{~min}$ after quenching from $\left.40^{\circ} \mathrm{C}\right) ; \phi_{\mathrm{MeOH}}=0.5$ and $c=4 \times 10^{-6} \mathrm{~g} \mathrm{~cm}^{-3}$. Solid and dotted curves, theoretical values for polydisperse spheres fitted to the data at 15 and $40^{\circ} \mathrm{C}$, respectively (see the main text).

PCF indicates that the solutions may be in the biphasic region, and the large particles are the concentrated droplet phases separated from the dilute solutions.

For dilute solutions of polydisperse spheres, $\left(K c / R_{\theta}\right)^{1 / 2}$ can be calculated by ${ }^{17}$

$$
\frac{R_{\theta}}{K c}=\frac{12 \pi N_{\mathrm{A}} c_{\mathrm{c}}}{k^{3}} \int_{0}^{\infty} \frac{[\sin (k R)-k R \cos (k R)]^{2}}{(k R)^{3}} w(R) \mathrm{d} R
$$


where $R$ is the radius of the sphere, $w(R)$ is the weight fraction of a sphere of radius $R, c_{\mathrm{c}}$ is the polymer mass concentration in the spherical concentrated phase, and $N_{\mathrm{A}}$ is the Avogadro constant. Assuming that the size distribution $w(R)$ obeys the log-normal distribution, ${ }^{17}$ equation (1) was fitted to the experimental results to obtain the weight-average radius $R_{\mathrm{w}}$, the ratio of $R_{\mathrm{w}}$ to the numberaverage $R_{\mathrm{n}}$ of the droplet phase and $c_{\mathrm{c}}$. The solid and dotted curves in Figure 1 show the fitting results, and the parameters obtained are listed in columns 5-8 in Table 1. Droplet sizes are on order of $100 \mathrm{~nm}$. The concentrations of the droplet phases are as high as $0.6 \mathrm{~g} \mathrm{~cm}^{-3}$ for both fractions and almost independent of temperature. The results of $c_{\mathrm{c}}$ for fractions PC5O5 and PCF indicate the similar affinities of the chiral and achiral monomer units to the mixed solvent.

\section{Circular dichroism induction}

None of the copolymer fractions exhibited CD in dilute THF solution, as in the case of the chiral homopolymer $\mathrm{PCF},{ }^{17}$ which indicates that the intramolecular chiral interaction between the main and side chains of the copolymers is too weak to energetically discriminate the rightand left-handed helical conformations in the fluorene main chain.

Figure 2 shows UV-visible absorption and CD spectra of the phase separating solution of PC5O5 quenched from 40 to $15^{\circ} \mathrm{C}$. In Panel a, the main peak of the UV-visible absorption, arising from the $\pi-\pi^{*}$ transition in the fluorene main chain, is essentially unchanged over time since quenching, but the peak height decreases, the peak wavelength slightly increases, and a new side-peak appears at $426 \mathrm{~nm}$. On the other hand, a bisign CD signal is induced in the fluorene main-chain absorption region by quenching in Panel $b$, just as in the case of fraction PCF reported previously. ${ }^{17}$ The induced CD disappeared upon heating to $40^{\circ} \mathrm{C}$ and reappeared by quenched again to $15^{\circ} \mathrm{C}$, which is similar to PCF and demonstrates the thermal reversibility of the $\mathrm{CD}$ induction. The phase-separating solutions were verified to have no optical anisotropy, ${ }^{17}$ which means that the induced $\mathrm{CD}$ does not arise from a liquid crystal phase. The polyfluorene chain is known to take $5 / 2$ or $5 / 1$ helical conformations favorably ${ }^{14,16}$ so that the CD induction indicates uneven population of the right- and lefthanded helical conformations of the copolymer main-chain. The Kuhn dissymmetry factor $g_{\mathrm{c}} \equiv \Delta \varepsilon / \varepsilon$ at $410 \mathrm{~nm}$ (the CD peak position) reaches the asymptotic value at approximately $60 \mathrm{~min}$ (cf. Insert of Figure 1), which is slightly faster than $g_{\mathrm{c}}$ of the PCF solution. ${ }^{17}$

The new side peak at $426 \mathrm{~nm}$ in Panel a corresponds to the so-called ' $\beta$-phase' observed for poly $(9,9$-di- $n$-octylfluorene $)$ films,${ }^{24,25}$ which is assigned to an almost planar conformation of the fluorene main chain with the torsional angle $\approx 160^{\circ}$ stabilized by the $n$-octyl side chains. ${ }^{26}$ A more pronounced side-peak was observed in the methanol-added THF solution of fraction $\mathrm{PC} 2 \mathrm{O} 8$, indicating that some of achiral monomer units take similar planer conformations in the concentrated droplet phase. Interestingly, a side-peak also grows in the CD spectrum (Panel b) at the same wavelength, which may arise from the achiral monomer unit in the copolymer chain taking the ' $\beta$-phase' conformation.

In the methanol-added THF solution with the same $\phi_{\mathrm{MeOH}}$ and $c$, a similar CD induction was observed for PC6O4 quenched from 40 to $15^{\circ} \mathrm{C}$, but a CD signal appeared for PC2O 8 even at $40^{\circ} \mathrm{C}$ and did not change with time after quenching to $15^{\circ} \mathrm{C}$. The $\mathrm{CD}$ induction experiments were examined twice for fraction PC2O8, and nearly the same CD spectra were obtained, confirming the reproducibility of the $\mathrm{CD}$ induction for this fraction.

Figure 3 shows the asymptotic CD absorption spectra for all chiralachiral random copolymers and the chiral homopolymer PCF in methanol-added THF solutions $\left(\phi_{\mathrm{MeOH}}=0.5, c=4 \times 10^{-7} \mathrm{~g} \mathrm{~cm}^{-3}\right)$.
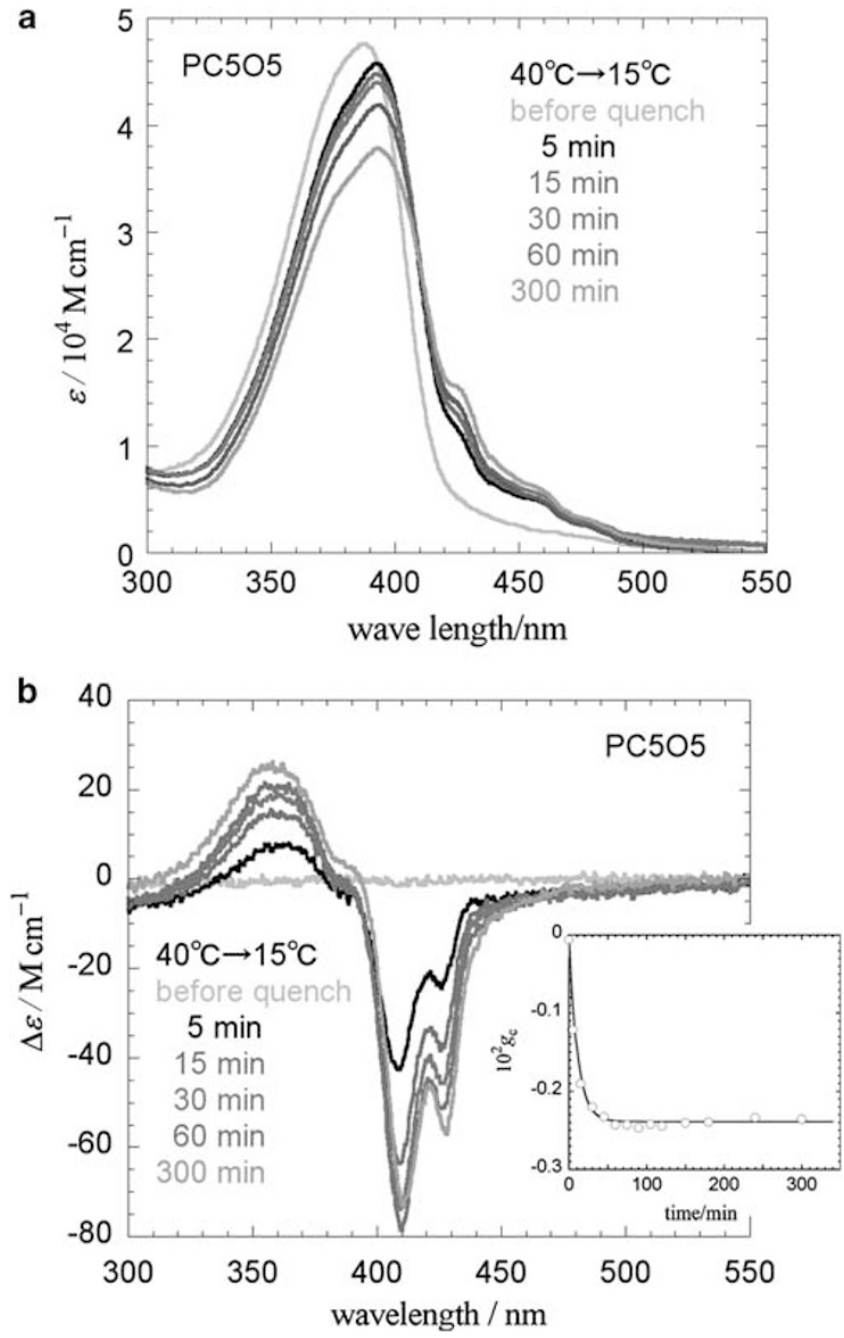

Figure 2 Spectra of the molar circular dichroism $\Delta \varepsilon$ (a) and the average molar extinction coefficient $\varepsilon$ (b) for methanol-added THF solution of fraction PC505; inset in Panel $\mathbf{b}$ : time dependence of the Kuhn dissymmetry factor at $410 \mathrm{~nm}$. A full color version of this figure is available at Polymer Journal online.

The bisign CD main signals induced in PCF and PC2O8 solutions are positive in the longer wavelength region but opposite in $\mathrm{PC6O} 4$ and PC5O5 solutions, and $\mathrm{PC} 8 \mathrm{O} 2$ exhibits almost no CD. The sign of the side peak of PC2O8 (at $429 \mathrm{~nm}$ ) is also opposite to that of PC5O5 (at $426 \mathrm{~nm}$ ). The insert in Figure 3 shows the $x$ dependence of the Kuhn dissymmetry factor at the CD peak in the region of $400-420 \mathrm{~nm}$. These results demonstrate that the helical screw sense of the chiralachiral random copolymer is inverted twice with changing $x$. To the best of our knowledge, this is the first observation of the double sense inversions in helical chiral-achiral random copolymers.

\section{DISCUSSION}

Consider the origin of the double sense inversions in helical chiralachiral random copolymers. For simplicity, assume that the mainchain bond (for example the internal rotation angle) of the helical polymer chain takes P- or M-states (a two-state model). The P-helix (M-helix) means the sequence of main-chain bonds taking the $\mathrm{P}$-state (M-state). In a concentrated solution, a bond taking the P-state (M-state) feels the chiral molecular field $\bar{w}^{*}(\mathrm{P})\left(\bar{w}^{*}(\mathrm{M})\right)$ generated by neighboring polymer molecules. ${ }^{27}$ If the solution contains $\mathrm{P}$ - and 


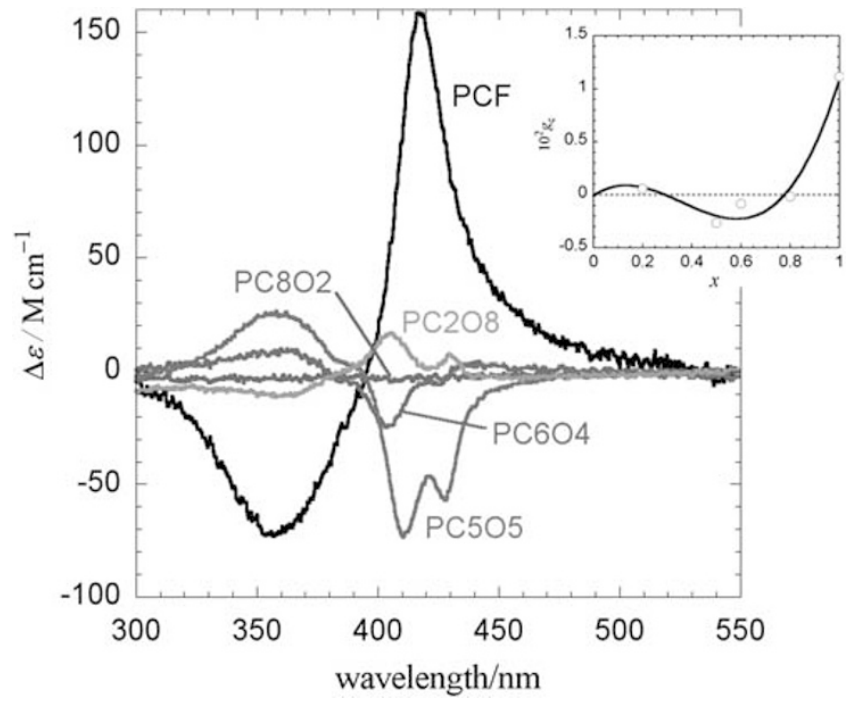

Figure 3 Asymptotic CD absorption spectra for all chiral-achiral random copolymers and the chiral homopolymer PCF in methanol-added THF solutions; inset: the $x$ dependence of the Kuhn dissymmetry factor at the $C D$ peak in the region of $400-420 \mathrm{~nm}$. A full color version of this figure is available at Polymer Journal online.

M-state bonds of fractions $f_{\mathrm{P}}$ and $f_{\mathrm{M}}$, respectively, the molecular fields, $\bar{w}^{*}(\mathrm{P})$ and $\bar{w}^{*}(\mathrm{M})$, may be given by

$$
\bar{w}^{*}(\mathrm{P})=w_{\mathrm{PP}}^{*} f_{\mathrm{P}}+w_{\mathrm{PM}}^{*} f_{\mathrm{M}}, \bar{w}^{*}(\mathrm{M})=w_{\mathrm{PM}}^{*} f_{\mathrm{P}}+w_{\mathrm{MM}}^{*} f_{\mathrm{M}}
$$

where $w_{\mathrm{PB}}^{*} w_{\mathrm{MM}}^{*}$, and $w_{\mathrm{PM}}^{*}$ are the chiral interactions between two pairs of adjacent monomer units connected by the bonds both taking the P-state, both taking the M-state, and taking the P- and M-states, respectively. Because the molecular field may include both enthalpic and entropic contributions, $\bar{w}^{*}(\mathrm{P})-\bar{w}^{*}(\mathrm{M})$ can be regarded as the free energy difference $2 \Delta G_{\mathrm{h}}$ of a bond when taking the P-state and M-state in the concentrated solution. From equation (2), $\Delta G_{\mathrm{h}}$ is a linear function of the enantiomer excess $2 f_{\mathrm{P}}-1$, for example, $\Delta G_{\mathrm{h}}=\kappa\left(2 f_{\mathrm{P}}-1\right)$ $+\lambda$ where $\kappa$ and $\lambda$ are parameters related to the chiral interactions. In the previous study, ${ }^{17}$ it was assumed that $\lambda=0$, but $w^{*}$ PP and $w^{*} \mathrm{MM}$ are not necessarily identical unless the interacting monomer units are both achiral.

In the case of a chiral-achiral random copolymer solution, $\Delta G_{\mathrm{h}}$ may depend on the kinds of adjacent monomer units connected by the bond under consideration: ${ }^{28}$

$$
\begin{aligned}
\Delta G_{\mathrm{h}, \mathrm{CC}} & =\kappa_{\mathrm{CC}}\left(2 f_{\mathrm{P}}-1\right)+\lambda_{\mathrm{CC}}, \\
\Delta G_{\mathrm{h}, \mathrm{CA}} & =\kappa_{\mathrm{CA}}\left(2 f_{\mathrm{P}}-1\right)+\lambda_{\mathrm{CA}}, \\
\Delta G_{\mathrm{h}, \mathrm{AA}} & =\kappa_{\mathrm{AA}}\left(2 f_{\mathrm{P}}-1\right)
\end{aligned}
$$

where the subscripts $\mathrm{C}$ and A denote the chiral and achiral monomer units, respectively. Because the achiral homopolymer should be racemic at $2 f_{\mathrm{P}}-1=0, \lambda_{\mathrm{AA}}$ must be zero. Helical polymers must have long sequences of the one-sense helical state along the main chain, or the helix reversal must be a rare event. Thus, the free energy $\Delta G_{\mathrm{r}}$ of the helix reversal, where the adjacent bonds take the opposite helical state, must be quite high. ${ }^{29}$ In what follows, the dependence of $\Delta G_{\mathrm{r}}$ on the kinds of adjacent monomer units connected by the bond under consideration is not considered, which should have a minor effect on the helical screw sense inversion.

The enantiomer excess $2 f_{\mathrm{P}}-1$ of the chiral-achiral random copolymer chain can be calculated by the matrix method for the Ising model. ${ }^{28,30,31}$ The matrix includes the statistical weights of the P- and
M-states and the helix reversal, which are respectively written as

$$
\left\{\begin{array}{l}
u_{\mathrm{P}, i(k) i(k+1)}=\exp \left(\Delta G_{\mathrm{h}, i(k) i(k+1)} / R T\right) \\
u_{\mathrm{M}, i(k) i(k+1)}=\exp \left(-\Delta G_{\mathrm{h}, i(k) i(k+1)} / R T\right) \\
v=\exp \left(-\Delta G_{\mathrm{r}} / R T\right)
\end{array}\right.
$$

where the subscripts $i(k)(1 \leqslant k \leqslant N)$, taking $\mathrm{C}$ (the chiral monomer unit)) or A (the achiral monomer unit), specify the copolymer sequence and $R T$ is the gas constant multiplied by the absolute temperature. (This $R$ should be distinguished from the radius $R$ in equation (1)) Generating 100 sequences of chiral-achiral random copolymers with a given $N$ and mole fraction $x$ of the chiral unit on a computer, $2 f_{\mathrm{P}}-1$ can be calculated numerically for the given values of $\Delta G_{\mathrm{h}, \mathrm{CC}}, \Delta G_{\mathrm{h}, \mathrm{CA}}, \Delta G_{\mathrm{h}, \mathrm{AA}}$, and $\Delta G_{\mathrm{r}}$ in the routine procedure.

The calculated $2 f_{\mathrm{P}}-1$ must fulfill equation (3) for $\Delta G_{\mathrm{h}, \mathrm{CC}}, \Delta G_{\mathrm{h}, \mathrm{CA}}$, and $\Delta G_{\mathrm{h}, \mathrm{AA}}$. This self-consistent calculation can be performed as follows. From equation (3), the relations among $\Delta G_{\mathrm{h}, \mathrm{CC}}, \Delta G_{\mathrm{h}, \mathrm{CA}}$, and $\Delta G_{\mathrm{h}, \mathrm{AA}}$ are obtained:

$$
\begin{gathered}
\Delta G_{\mathrm{h}, \mathrm{CA}}=\frac{\kappa_{\mathrm{CA}}}{\kappa_{\mathrm{CC}}}\left(\Delta G_{\mathrm{h}, \mathrm{CC}}-\lambda_{\mathrm{CC}}\right)+\lambda_{\mathrm{CA}}, \\
\Delta G_{\mathrm{h}, \mathrm{AA}}=\frac{\kappa_{\mathrm{AA}}}{\kappa_{\mathrm{CC}}}\left(\Delta G_{\mathrm{h}, \mathrm{CC}}-\lambda_{\mathrm{CC}}\right)
\end{gathered}
$$

A trial value of $\Delta G_{\mathrm{h}, \mathrm{CC}}$ is chosen first, and $\Delta G_{\mathrm{h}, \mathrm{CA}}$ and $\Delta G_{\mathrm{h}, \mathrm{AA}}$ are calculated from equation (5) using a given set of the parameters $\kappa_{\mathrm{CA}} / \kappa_{\mathrm{CC}}, \kappa_{\mathrm{AA}} / \kappa_{\mathrm{CC}}, \lambda_{\mathrm{CC}}$, and $\lambda_{\mathrm{CA}}$. Then, $2 f_{\mathrm{P}}-1$ is calculated by the matrix method using those values of $\Delta G_{\mathrm{h}}$ and a given value of $\Delta G_{\mathrm{r}}$ and the resulting $2 f_{\mathrm{P}}-1$ is substituted into the first equation of equation (3) to check the equality. The self-consistent value of $\Delta G_{\mathrm{h}, \mathrm{CC}}$ that fulfills the equation is sought.

The previous result indicated that $2 f_{\mathrm{P}}-1$ for PCF in the phaseseparating solution is close to zero at $40^{\circ} \mathrm{C}$ but takes a positive finite value at $15^{\circ} \mathrm{C} .{ }^{17}$ To reproduce these results, $\kappa_{\mathrm{CC}}=40 \mathrm{~J} \mathrm{~mol}^{-1}$ and $\lambda_{\mathrm{CC}}=2 \mathrm{~J} \mathrm{~mol}^{-1}$ were selected (using these parameters, $2 f_{\mathrm{P}}-1$ for PCF becomes 0.09 at $40{ }^{\circ} \mathrm{C}$ and 0.38 at $15^{\circ} \mathrm{C}$ ). Furthermore, $N=220$ (the average value of our five polymer fractions) and $\Delta G_{\mathrm{r}}=10 \mathrm{~kJ} \mathrm{~mol}^{-1}$ (a typical value for helical polymers such as polyacetylene or polyisocyanate derivatives ${ }^{32,33}$ ). The remaining parameters in equation (2), $\kappa_{\mathrm{CA}}, \kappa_{\mathrm{AA}}$, and $\lambda_{\mathrm{CA}}$, were taken as adjustable parameters. (The $\kappa$ and $\lambda$ parameters may depend on the polymer concentration $c_{\mathrm{c}}$ in the concentrated phase, but here it is assumed that these parameters are independent of $x$ because $c_{\mathrm{c}}$ may be insensitive to $x$ due to similar affinities of the chiral and achiral monomer units to the mixed solvent, mentioned above.).

Figure 4 shows the results of $2 f_{\mathrm{P}}-1$ at $15^{\circ} \mathrm{C}$ as a function of $\Delta G_{\mathrm{h}, \mathrm{CC}}$ and $x$ for $\kappa_{\mathrm{CA}} / \kappa_{\mathrm{CC}}=-2.5, \kappa_{\mathrm{AA}} / \kappa_{\mathrm{CC}}=3.5, \lambda_{\mathrm{CC}}=2 \mathrm{~J} \mathrm{~mol}^{-1}$, and $\lambda_{\mathrm{CA}}=-4 \mathrm{~J} \mathrm{~mol}^{-1}$. Slight fluctuations in the calculated $2 f_{\mathrm{P}}-1$ values arise from statistical errors in the chiral monomer content generated. The straight line in Figure 4 represents the linear relation of $2 f_{\mathrm{P}}-1=\left(\Delta G_{\mathrm{h}}-\lambda_{\mathrm{CC}}\right) / \kappa_{\mathrm{CC}}$ with $\kappa_{\mathrm{CC}}=40 \mathrm{~J} \mathrm{~mol}^{-1}$. The intersecting point of this line and the curve for each $x$ fulfills equation (3). At large $x$, the curve intersects with the line once, but, in the small $x$ region, there are three intersecting points.

Figure 5 shows the self-consistent solution of $2 f_{\mathrm{P}}-1$ at $15^{\circ} \mathrm{C}$, obtained from Figure 4, as a function of $x$. At large $x(>0.227)$, the calculation gives the unique self-consistent solution of $2 f_{\mathrm{p}}-1$. When $x$ decreases from unity, $2 f_{\mathrm{P}}-1$ changes from positive to negative (the solution of branch 1), corresponding to the first helical screw sense inversion. However, one negative and two positive solutions appear at $x<0.227$. Among these solutions, the positive one reaching the origin (labeled as the branch 2) should be the real solution because the achiral homopolymer must be racemic. Therefore, a transition 


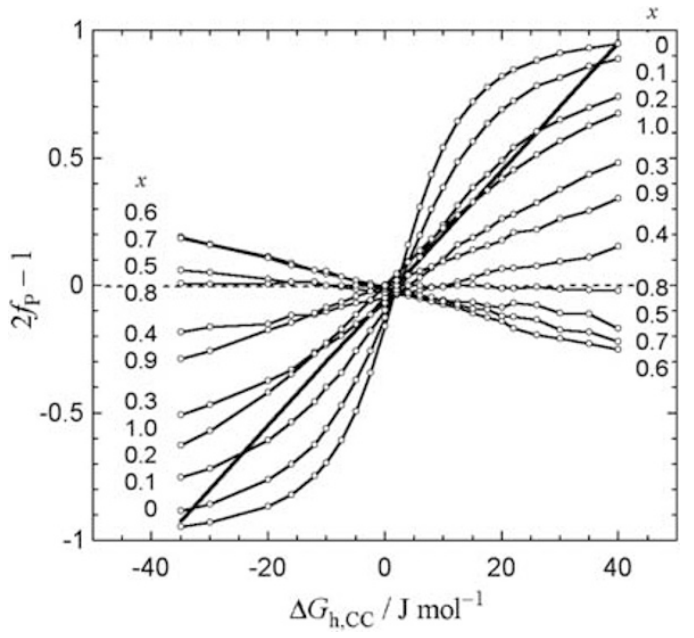

Figure 4 Enantiomer excess as a function of $\Delta G_{h, c c}$ and $x$, calculated by the matrix method for the Ising model of the random copolymer with $\kappa_{\mathrm{CA}} / \kappa_{\mathrm{CC}}=-2.5, \quad \kappa_{\mathrm{AA}} / \kappa_{\mathrm{CC}}=3.5, \quad \lambda_{\mathrm{CC}}=2 \mathrm{~J} \mathrm{~mol}^{-1}, \quad \lambda_{\mathrm{CA}}=-4 \mathrm{~J} \mathrm{~mol}^{-1}$, $\Delta G_{\mathrm{r}}=10 \mathrm{~kJ} \mathrm{~mol}^{-1}, N=220$, and $T=288 \mathrm{~K}\left(15^{\circ} \mathrm{C}\right)$.

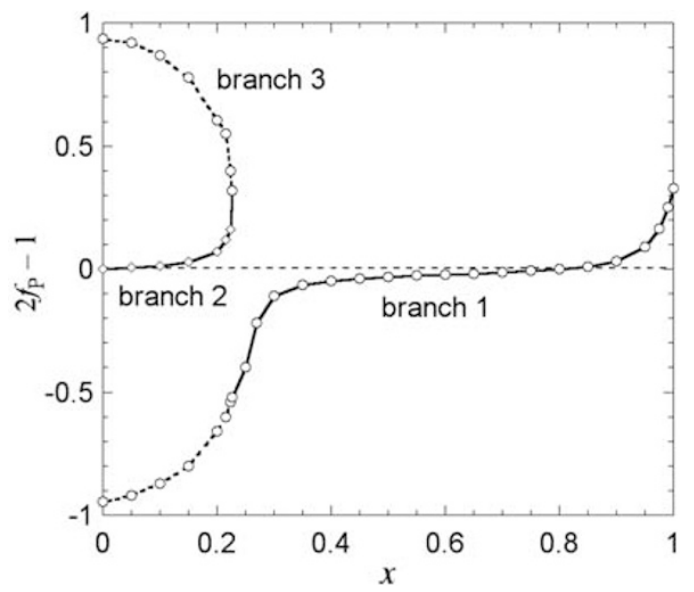

Figure 5 Self-consistent solutions of the enantiomer excess $2 f_{\mathrm{p}}-1$ as a function of $x$ for the parameters listed in the text.

from branch 1 to 2 is expected with decreasing $x$, which corresponds to the second helical screw sense inversion. This discontinuous transition is an interesting phenomenon. The CD induction at $x$ between 0.2 and 0.5 will be studied experimentally in more detail in the near future.

\section{CONCLUSIONS}

Methanol-added THF solutions of chiral-achiral random copolymers of fluorene derivatives with different chiral monomer content $x$ were studied. The addition of methanol to dilute THF solutions of the copolymers and the chiral homopolymer induced a liquid-liquid phase separation that produced concentrated phase droplets with a polymer concentration as high as $0.6 \mathrm{~g} \mathrm{~cm}^{-3}$ and a size of the order of $100 \mathrm{~nm}$. In the concentrated phase, the copolymers and homopolymer exhibited mostly thermo-reversible circular dichroism (CD) induction after quenching in the main-chain fluorene absorption region, demonstrating the uneven population of the right- and left-handed helical conformation in the polymer chains. From the sign of the induced CD, two helical screw-sense inversions were found by changing $x$. The Ising model for chirally interacting chiral-achiral random copolymers can explain the double screw-sense inversions.

\section{CONFLICT OF INTEREST}

The authors declare no conflict of interest.

\section{ACKNOWLEDGEMENTS}

Y Sanada thanks the Global Center of Excellence Program, the 'Global Education and Research Center for Bio-Environmental Chemistry' of Osaka University. This work was partly supported by a Grant in Aid for Scientific Research on Priority Area 'Soft Matter Physics.'

1 Burroughes, J. H., Bradley, D. D. C., Brown, A. R., Marks, R. N., Mackay, K., Friend, R. H., Burns, P. L. \& Holmes, A. B. Light-emitting diodes based on conjugated polymers. Nature 347, 539-541 (1990)

2 Scherf, U. \& Neher, D. (eds). Polyfluorenes. Adv. Polym. Sci., Springer: Berlin, Heiderberg, (2008)

3 Grimsdale, A. C. \& Müllen, K. Polyphenylene-type emissive materials: poly(paraphenylene)s, polyfluorenes, and ladder polymers. Adv. Polym. Sci. 199, 1-82 (2006).

4 McQuade, D. T., Pullen, A. E. \& Swager, T. M. Conjugated polymer-based chemical sensors. Chem. Rev. 100, 2537-2574 (2000).

5 Oda, M., Nothofer, H.- G., Lieser, G., Scherf, U., Meskers, S. C. J. \& Neher, D. Circularly polarized electroluminescence from liquid-crystalline chiral polyfluorenes. Adv. Mater. 12, 362-365 (2000).

6 Oda, M., Meskers, S. C. J., Nothofer, H. G., Scherf, U. \& Neher, D. Chiroptical properties of chiral-substituted polyfluorenes. Synth. Met. 111-112, 575-577 (2000).

7 Tang, H.- Z., Fujiki, M. \& Sato, T. Thermodriven Conformational Transition of Optically Active Poly[2,7-\{9,9-bis[(S)-2-methyloctyl]\}fluorene] in Solution. Macromolecules. 35, 6439-6445 (2002).

8 Wu, L., Sato, T., Tang, H.- Z. \& Fujiki, M. Conformation of a polyfluorene derivative in solution. Macromolecules. 37, 6183-6188 (2004).

9 Wu, L. \& Sato, T. Aggregation-induced circular dichroism of optically active polyfluorene derivatives in solution. Kobunshi. Ronbunshu. 63, 505-511 (2006).

10 Langeveld-Voss, B. M. W., Waterval, R. J. M., Janssen, R. A. J. \& Meijer, E. W. Principles of 'Majority Rules' and 'Sergeants and Soldiers' Applied to the Aggregation of Optically Active Polythiophenes: Evidence for a Multichain Phenomenon. Macromolecules. 32, 227-230 (1999).

11 Nakashima, H., Fujiki, M., Koe, J. R. \& Motonaga, M. Solvent and Temperature Effects on the Chiral Aggregation of Poly(alkylarylsilane)s Bearing Remote Chiral Groups. J. Am. Chem. Soc. 123, 1963-1969 (2001).

12 Goto, H. \& Yashima, Y. Electron-induced switching of the supramolecular chirality of optically active polythiophene aggregates. J. Am. Chem. Soc. 124, 7943-7949 (2002).

13 Peng, W., Motonaga, M. \& Koe, J. R. Chirality control in optically active polysilane aggregates. J. Am. Chem. Soc. 126, 13822-13826 (2004).

14 Lieser, G., Oda, M., Miteva, T., Meisel, A., Nothofer, H.- G., Scherf, U. \& Neher, D. Ordering, graphoepitaxial orientation, and conformation of a polyfluorene derivative of the 'hairly-rod' type on an oriented substrate of polyimide. Macromolecules 33, 4490-4495 (2000).

15 Knaapila, M. \& Winokur, M. J. Structure and Morphology of Polyfluorenes in Solutions and the Solid State. Adv. Polym. Sci. 212, 227-272 (2008).

16 Hong, S. Y., Kim, D. Y., Kim, C. Y. \& Hoffmann, R. Origin of the broken conjugation in m-phenylene linked conjugated polymers. Macromolecules. 34, 6474-6481 (2001).

17 Sanada, Y. \& Sato, T. Induced circular dichroism of an optically active polyfluorene derivative in phase-separating solutions. Polym. J. 42, 195-200 (2010).

18 Green, M. M., Reidy, M. P., Johnson, R. D., Darling, G., O'Leary, D. J. \& Wilson, G. Macromolecular Stereochemistry: the out-of-proportion influence of optically active comonomers on the conformational characteristics of polyisocyanates: the sergents \& soldiers experiment. J. Am. Chem. Soc. 111, 6452-6454 (1989).

19 Green, M. M., Peterson, N. C., Sato, T., Teramoto, A., Cook, R. \& Lifson, S. A Helical polymer with cooperative response to chiral information. Science 268, 1860-1866 (1995).

20 Green, M. M., Park, J. W., Sato, T., Teramoto, A., Lifson, S., Selinger, R L B. \& Selinger, J. V. The macromolecular route to chiral amplification. Angew. Chem. Int. Ed. 38, 3138-3154 (1999).

21 Maeda, K. \& Okamoto, Y. Synthesis and conformational characteristics of poly(phenyl isocyanate)s bearing an optically active ester group. Macromolecules. 32, 974-980 (1999).

22 Koe, J. R., Fujiki, M., Motonaga, M. \& Nakashima, H. Cooperative helical order in optically active poly(diarylsilylenes). Macromolecules 34, 1082-1089 (2001).

23 Matsuda, Y., Biyajima, Y. \& Sato, T. Thermal denaturation, renaturation, and aggregation of a double-helical polysaccharide xanthan in aqueous solution. Polym. J. 41, 526-532 (2009).

24 Grell, M., Bradley, D D C., Ungar, G., Hill, J. \& Whitehead, K. S. Interplay of physical structure and photophysics for a liquid crystalline polyfluorene. Macromolecules $\mathbf{3 2}$, 5810-5817 (1999). 
25 Knaapila, M. \& Winokur, M. J. Structure and morphology of polyfluorenes in solutions and the solid state. Adv. Polym. Sci. 212, 227-272 (2008).

26 Chunwaschirasiri, W., Tanto, B., Huber, D. L. \& Winokur, M. J. Chain conformations and photoluminescence of poly(di-n-octylfluorene). Phys. Rev. Lett. 94, 107402 (2005).

27 Baumgarten, J. L. Ferrochirality: a simple theoretical model of interacting dynamically invertible helical polymers, 1 the basic effects. Macromol. Rapid. Commun. 15, 175-182 (1994).

28 Sato, T., Terao, K., Teramoto, A. \& Fujiki, M. On the composition-driven helical screw-sense inversion of chiral-achiral random copolymers. Macromolecules 35, 5355-5357 (2002).

29 Lifson, S., Andreola, C., Peterson, N. C. \& Green, M. M. A Statistical thermodynamic analysis of the cooperative source of helix sense preference in polyisocyanates: the amplification of a conformational equilibrium deuterium isotope effect. J. Am. Chem. Soc. 111, 8850-8858 (1989).

$30 \mathrm{Gu}$, H., Sato, T., Teramoto, A., Varichon, L. \& Green, M. M. Molecular mechanisms for the optical activities of polyisocyanates induced by intramolecular chiral perturbations. Polym. J. 29, 77-84 (1997).

$31 \mathrm{Gu}$, H., Nakamura, Y., Sato, T., Teramoto, A., Green, M. M., Jha, S. K., Andreola, C. \& Reidy, M. P. Optical rotation of random copolyisocyanates of chiral adn achiral monomers: sergeant adn soldier copolymers. Macromolecules 31, 6362-6368 (1998).

32 Morino, K., Maeda, K., Okamoto, Y., Yashima, E. \& Sato, T. Temperature dependence of helical structures of poly(phenylacetylene) derivatives bearing an optically active substituent. Chem. Eur. J. 8, 5112-5120 (2002).

33 Sato, T., Terao, K., Teramoto, A. \& Fujiki, M. Molecular properties of helical polysilylenes in solution. Polymer 44, 5477-5495 (2003). 